To appear in The Royal Society of Edinburgh Proc. A (Mathematics)

\title{
THE LITTLEWOOD-PALEY-RUBIO DE FRANCIA PROPERTY OF A BANACH SPACE FOR THE CASE OF EQUAL INTERVALS
}

\author{
T. P. HYTÖNEN ${ }^{1}$, J. L. TORREA ${ }^{2}$, AND D. V. YAKUBOVICH ${ }^{2}$
}

\section{INTRODUCTION AND RESULTS}

Let $X$ be a Banach space, with the norm denoted as $|\cdot|_{X}$. Put $\mathbf{T}=[0,1)$, which we identify with the additive group $\mathbb{R} / \mathbb{Z}$. Then the characters of $\mathbf{T}$ are the functions $e_{k}(t)=e^{i 2 \pi k t}, k \in \mathbb{Z}$. Let $L_{X}^{p}(\mathbf{T})$ be the Bochner space of $X$-valued $p$-integrable functions on $\mathbf{T}$. For intervals $I \subset \mathbb{Z}$, the corresponding partial sums of the Fourier series of $f$ are denoted by

$$
S_{I} f:=\sum_{k \in I} \hat{f}(k) e_{k} .
$$

In the case when $X=\mathbb{C}$, useful inequalities of the type

$$
\left\|\left(\sum_{j}\left|S_{I_{j}} f\right|^{2}\right)^{1 / 2}\right\|_{L^{p}(\mathbf{T})} \leq C\|f\|_{L^{p}(\mathbf{T})}
$$

are well known. When $\left\{I_{j}\right\}_{j \in \mathbb{Z}}$ is the collection of dyadic intervals, i.e., $I_{0}=\{0\}$ and $I_{j}=\operatorname{sgn}(j)\left[2^{|j|-1}, 2^{|j|}\right)$ for $|j|>0$, the estimate $(1.2)$ is the classical Littlewood-Paley inequality which is valid (as well as the reverse estimate with $\geq$ in place of $\leq$ ) for all $p \in(1, \infty)$. If the $I_{j}$ are disjoint intervals of equal length, then (1.2) holds if and only if $p \in[2, \infty)$; this was first proved by L. Carleson [6], and then in different ways by both A. Córdoba [7] and J. L. Rubio de Francia [19], who also finally showed that, for the same range of exponents, the analogue of (1.2) for the case of the real line is actually true for an arbitrary collection of disjoint subintervals $I_{j}$ of $\mathbb{R}$, see [20]. As we will explain later, the versions of (1.2) for the unit circle and for the real line are equivalent.

By Hinčin's inequality, there is a two-sided comparison

\footnotetext{
${ }^{1}$ The first author is supported by the Academy of Finland, project 114374 "Vector-valued singular integrals".

${ }^{2}$ The second and the third authors acknowledge the support of the Grant MTM2005-08359-C03-01 by the European Fund FEDER and Ministry of Science and Technology of Spain (MEC). The third author also acknowledges the support of MEC under the Ramón y Cajal Programme (2002).
}

Date: November 13, 2018. 


$$
\left(\sum_{j}\left|a_{j}\right|^{2}\right)^{1 / 2} \bar{\sim}_{p}\left(\mathbb{E}\left|\sum_{j} \mathbf{r}_{j} a_{j}\right|^{p}\right)^{1 / p}
$$

for all $0<p<\infty$, where the $\mathbf{r}_{j}$ are independent Rademacher random variables on some probability space $\Omega$ (i.e., they take the values +1 and -1 with equal probability $1 / 2$ ), and $\mathbb{E}$ is the mathematical expectation. Thus, an equivalent formulation of (1.2) reads

$$
\left\|\mathbb{E}\left|\sum_{j} \mathbf{r}_{j} S_{I_{j}} f\right|\right\|_{L^{p}(\mathbf{T})} \leq C\|f\|_{L^{p}(\mathbf{T})} .
$$

This has proven to be a useful formulation when looking for analogues of the LittlewoodPaley type estimates (1.2) in the Bochner spaces $L_{X}^{p}(\mathbf{T})$. In particular, it is known from the work of J. Bourgain [4] that

$$
\left\|\mathbb{E}\left|\sum_{j} \mathbf{r}_{j} S_{I_{j}} f\right|_{X}\right\|_{L^{p}(\mathbf{T})} \leq C\|f\|_{L_{X}^{p}(\mathbf{T})}
$$

holds for the dyadic system of intervals if and only if $p \in(1, \infty)$ and $X$ is a Banach space with the unconditionality property of martingale differences (UMD). In fact, already the uniform boundedness of the partial sum projections (1.1) on $L_{X}^{p}(\mathbf{T})$ is equivalent to $X \in U M D$, since both are equivalent to the boundedness of the Hilbert transform $H=$ $-i\left[S_{[0, \infty)}-S_{(-\infty, 0)}\right]$ on $L_{X}^{p}(\mathbf{T})$; cf. [3, 4, [5].

As for vector-valued analogues of (1.2) for other systems of intervals, E. Berkson, T. A. Gillespie and J. L. Torrea [1] have introduced the following terminology. The space $X$ is said to have the $L P R_{p}$ (Littlewood-Paley-Rubio) property if (1.3) holds for an arbitrary finite disjoint collection of intervals $I_{j}$ with a constant independent of the intervals and $f \in L_{X}^{p}(\mathbf{T})$. To be precise, the corresponding property in [1] was considered in the context of $L_{X}^{p}(\mathbb{R})$ that is

$$
\left\|\mathbb{E}\left|\sum_{j} \mathbf{r}_{j} S_{J_{j}}^{\mathbb{R}} f\right|_{X}\right\|_{L^{p}(\mathbb{R})} \leq C\|f\|_{L_{X}^{p}(\mathbb{R})}
$$

for disjoint intervals $J_{j} \subset \mathbb{R}$. Here

$$
S_{J_{j}}^{\mathbb{R}} f(x)=\int_{I_{j}} \hat{f}(\lambda) e^{i 2 \pi \lambda x} \mathrm{~d} \lambda
$$

where

$$
\hat{f}(\lambda)=\int_{\mathbb{R}} f(x) e^{-i 2 \pi \lambda x} \mathrm{~d} x
$$

is the Fourier transform of $f$. It was pointed out in [11] that this is equivalent to $L P R_{p}$ as defined by (1.3).

From the scalar-valued results and a restriction to a one-dimensional subspace it is clear that property $L P R_{p}$ is meaningful only for $p \in[2, \infty)$. Moreover, by using the characterization of UMD by the uniform $L_{X}^{p}(\mathbf{T})$-boundedness of the $S_{I}$, it follows that $L P R_{p} \Rightarrow U M D$. 
There is also a connection to the type of the Banach space, which is defined as follows. The space $X$ has type $t$ if and only if

$$
\mathbb{E}\left|\sum_{j} \mathbf{r}_{j} x_{j}\right|_{X} \leq C\left(\sum_{j}\left|x_{j}\right|_{X}^{t}\right)^{1 / t} .
$$

This condition is trivial (by the triangle inequality) for $t=1$; it becomes more restrictive with increasing $t$ and an impossibility for $t>2$. One defines

$$
p(X):=\sup \{t \in[1,2]: X \text { has type } t\} .
$$

It was shown in [1] that

$$
X \in L P R_{p} \quad \Rightarrow \quad p(X)=2 .
$$

Besides these observations, the $L P R_{p}$ property remains quite mysterious. For instance, it is not known whether there is an implication between $L P R_{p}$ and $L P R_{q}$ for two different $p, q \in[2, \infty)$, and basically the only known examples of spaces with this property are classical $L^{p}$ spaces. On the other hand, the $L P R_{p}$ property has some useful implications concerning multipliers of $X$-valued Fourier series, cf. [18, 12]. We refer to [8, 16] for related results.

In this note, our aim is to gain understanding of this property by studying the special case of equal-length intervals. Thus we say that the Banach space $X$ has the $L P R_{p}^{=}(\mathbf{T})$ (respectively $L P R_{p}^{=}(\mathbb{R})$ ) property if (1.3) (respectively (1.4)) holds with a fixed constant $C$ for all $f \in L_{X}^{p}(\mathbf{T})$ (respectively $f \in L_{X}^{p}(\mathbb{R})$ ) and all finite sequences of non-overlapping intervals $I_{j}$ of equal length. We also introduce a number of other variants of this condition after recalling some notation.

Let $w \in L^{1}(\mathbf{T})$ be a weight function. We denote by $L_{X}^{p}(\mathbf{T}, w)$ the weighted $L^{p}$ space, i.e., the $L^{p}$ space with respect to the measure $w(t) \mathrm{d} t$. In the sequel, we are particularly interested in weights in the Muckenhoupt $A_{q}$ classes.

Let $M:[0, \infty) \rightarrow[0, \infty)$ be a convex function such that $M(0)=0$ and $\lim _{t \rightarrow \infty} M(t)=$ $\infty$. Recall that the Orlicz space $L_{M}(\mathbf{T})$ consists of those functions $g \in L^{1}(\mathbf{T})$, which satisfy $\int_{\mathbf{T}} M(\lambda|g(t)|) \mathrm{d} t<\infty$ for some $\lambda>0$. It is a Banach space with the norm

$$
\|g\|_{L_{M}(\mathbf{T})}=\inf \left\{\lambda>0: \quad \int_{\mathbf{T}} M(|g(t)| / \lambda) \mathrm{d} t<1\right\} .
$$

Its $X$-valued version is denoted with the subscript $X$, as usual. In the case of the function $M_{0}(t)=\exp (t)-1$, we denote $L_{M_{0}}(\mathbf{T})=\exp L(\mathbf{T})$.

In the following conditions, it is always understood that there should be a fixed $C$ such that the given inequality holds whenever $I_{j}$ are allowed to be any finite collection of nonoverlapping intervals of equal length, and $f$ an arbitrary function in the space indicated by the right-hand side of the inequality. We say that $X$ satisfies $L P R_{p}^{=}(\mathbf{T}, w)$ if

$$
\left\|\mathbb{E}\left|\sum_{j} \mathbf{r}_{j} S_{I_{j}} f\right|_{X}\right\|_{L^{p}(\mathbf{T}, w)} \leq C\|f\|_{L_{X}^{p}(\mathbf{T}, w)},
$$


we say that $X$ satisfies $L P R_{\infty}^{=}(\mathbf{T})$ if

$$
\left\|\mathbb{E}\left|\sum_{j} \mathbf{r}_{j} S_{I_{j}} f\right|_{X}\right\|_{\exp L(\mathbf{T})} \leq C\|f\|_{L_{X}^{\infty}(\mathbf{T})}
$$

and finally $X$ satisfies $L P R_{\infty, 1}^{=}(\mathbf{T})$ if

$$
\left\|\mathbb{E}\left|\sum_{j} \mathbf{r}_{j} S_{I_{j}} f\right|_{X}\right\|_{L^{1}(\mathbf{T})} \leq C\|f\|_{L_{X}^{\infty}(\mathbf{T})}
$$

We shall also say that $X$ satisfies $\operatorname{LPR}_{p}^{=}(\mathbb{R}, w)$ if an analogue of formula (1.7) holds, with $\mathbf{T}$ replaced by $\mathbb{R}$.

Out main result is that all these equal-interval conditions are equivalent, and that moreover they admit a simple characterization in terms of well-established Banach space properties. This also sharpens the necessary conditions for the original $L P R_{p}$ property from the earlier results quoted above.

Theorem 1.1. (Case of T.) Let $X$ be a Banach space. The following conditions are equivalent:

- $X$ is a UMD space with type 2 ,

- $X$ has $L P R_{p}^{=}(\mathbf{T}, w)$ for all $p \in[2, \infty)$ and all $w \in A_{p / 2}$,

- $X$ has $L P R_{p}^{=}(\mathbf{T})$ for some $p \in[2, \infty)$,

- $X$ has $L P R_{\infty}^{=}(\mathbf{T})$,

- $X$ has $L P R_{\infty, 1}^{=}(\mathbf{T})$.

We also have the following result.

Theorem 1.2. (Case of $\mathbb{R}$.) Let $X$ be a Banach space. The following conditions are equivalent:

- $X$ is a UMD space with type 2 ,

- $X$ has $L P R_{p}^{=}(\mathbb{R}, w)$ for all $p \in[2, \infty)$ and all $w \in A_{p / 2}$,

- $X$ has $L P R_{p}^{=}(\mathbb{R})$ for some $p \in[2, \infty)$.

The proof of Theorem 1.1 will comprise the main part of our arguments. Since we have the following containments, with bounded inclusion maps,

$$
L^{\infty}(\mathbf{T}) \subset \exp L(\mathbf{T}) \subset L^{p}(\mathbf{T}) \subset L^{1}(\mathbf{T}), \quad p \in(1, \infty),
$$

it is immediate that $L P R_{p}^{=}(\mathbf{T}) \Rightarrow L P R_{\infty, 1}^{=}(\mathbf{T})$ for all $p \in[2, \infty]$. Hence it suffices to show that $L P R_{\infty, 1}^{=}(\mathbf{T})$ implies UMD and type 2, and UMD together with type 2 imply $L P R_{p}^{=}(\mathbf{T}, w)$ for all $p \in[2, \infty)$ and $w \in A_{p / 2}$, as well as $L P R_{\infty}^{=}(\mathbf{T})$.

Theorem 1.2 will be derived from Theorem [1.1. In [11, a proof is provided in order to see that property $L P R_{p}$ for $\mathbb{R}$ implies the property $L P R_{p}$ for $\mathbf{T}$. This proof can be adapted directly, and one gets that $L P R_{p}^{=}(\mathbb{R})$ implies $L P R_{p}^{=}(\mathbf{T})$. The converse will follow from Lemma 4.1.

Finally, in Section 5 we will comment on the validity of $L^{\infty}$-BMO results. 


\section{THE NECESSITY OF UMD AND TYPE 2}

Let us first deal with the UMD property. This already follows from (1.9) with an arbitrary single interval $I$; the estimate says that

$$
\left\|S_{I} f\right\|_{L_{X}^{1}(\mathbf{T})} \leq C\|f\|_{L_{X}^{\infty}(\mathbf{T})},
$$

which implies a similar inequality with the Hilbert transform $H=-i\left[S_{[0, \infty)}-S_{(-\infty, 0)}\right]$ in place of $S_{I}$. Using the description of the Hardy space $H_{X}^{1}(\mathbf{T})$ in terms of $L_{X}^{\infty}(\mathbf{T})$-atoms, it follows that $H$ is bounded from $H_{X}^{1}(\mathbf{T})$ to $L_{X}^{1}(\mathbf{T})$. This implies its boundedness on $L_{X}^{p}(\mathbf{T})$ by O. Blasco [2], which in turn implies UMD by Bourgain [3].

In what follows, we will use the following well-known result.

Lemma 2.1 (Kahane's contraction principle, see [17], Proposition 2.5). For any sequences $\left\{a_{n}\right\} \subset \mathbb{C},\left\{x_{n}\right\} \subset X$ and any $p \in[1, \infty)$,

$$
\mathbb{E}\left|\sum_{n} a_{n} \mathbf{r}_{n} x_{n}\right|_{X}^{p} \leq\left(2 \sup _{n}\left|a_{n}\right|\right)^{p} \cdot \mathbb{E}\left|\sum_{n} \mathbf{r}_{n} x_{n}\right|_{X}^{p}
$$

We turn to the type 2 property, starting from the following bootstrapping of the original estimate.

Lemma 2.2. Let $X$ have $L P R_{\infty, 1}^{=}(\mathbf{T})$. Let $\left\{I_{j k}\right\}_{j, k}$ be a two-parameter family of equallength intervals such that for every fixed $j$, the family $\left\{I_{j k}\right\}_{k}$ is finite and consists of disjoint intervals. Then

$$
\left\|\mathbb{E}\left|\sum_{j, k} \mathbf{r}_{j k} S_{I_{j k}} f_{j}\right|_{X}\right\|_{L^{1}(\mathbf{T})} \leq C\left\|\max _{\varepsilon_{j}= \pm 1}\left|\sum_{j} \varepsilon_{j} f_{j}\right|_{X}\right\|_{L^{\infty}(\mathbf{T})}
$$

for all finite families of $f_{j} \in L_{X}^{\infty}(\mathbf{T})$.

Proof. We may assume by approximation that the Fourier transforms $\hat{f}_{j}$ are compactly supported. Then we may choose intervals $J_{j}$ such that $J_{j} \supseteq \operatorname{supp} \hat{f}_{j}$, and also $J_{j} \supseteq I_{j k}$ for all $k$. Next we choose integers $N_{j}$ such that the translated intervals $J_{j}+N_{j}$ are all disjoint. Then, manipulating the random sums with the help of Kahane's contraction principle

$$
\begin{aligned}
& \left\|\mathbb{E}\left|\sum_{j, k} \mathbf{r}_{j k} S_{I_{j k}} f_{j}\right|_{X}\right\|_{L^{1}(\mathbf{T})}=\left\|\mathbb{E}\left|\sum_{j, k} \mathbf{r}_{j k} e_{-N_{j}} S_{I_{j k}+N_{j}} e_{N_{j}} f_{j}\right|_{X}\right\|_{L^{1}(\mathbf{T})} \\
& \leq\left. 2\left\|\mathbb{E}\left|\sum_{j, k} \mathbf{r}_{j k} S_{I_{j k}+N_{j}} e_{N_{j}} f_{j}\right|_{X}\right\|\right|_{L^{1}(\mathbf{T})}=2\left\|\mathbb{E}\left|\sum_{j, k} \mathbf{r}_{j k} S_{I_{j k}+N_{j}} \sum_{i} e_{N_{i}} f_{i}\right|_{X}\right\|_{L^{1}(\mathbf{T})} \\
& \leq 2 C\left\|\sum_{i} e_{N_{i}} f_{i}\right\|_{L_{X}^{\infty}(\mathbf{T})} \leq 4 C\left\|\max _{\varepsilon_{i}= \pm 1}\left|\sum_{j} \varepsilon_{j} f_{j}\right|_{X}\right\|_{L^{\infty}(\mathbf{T})},
\end{aligned}
$$

where, in the second equality, we used the fact that $S_{I_{j k}+N_{j}} e_{N_{i}} f_{i}=0$ if $j \neq i$. 
Corollary 2.3. Let $X$ have $L P R_{\infty, 1}^{=}(\mathbf{T})$. Let $x_{j} \in X$ and $\phi_{j} \in L^{\infty}(\mathbf{T})$ for $j=1, \ldots, N$. Then

$$
\left\|\mathbb{E}\left|\sum_{j=1}^{N} \mathbf{r}_{j}\left\|\phi_{j}\right\|_{L^{2}(\mathbf{T})} x_{j}\right|_{X}\right\|_{L^{1}(\mathbf{T})} \leq C\left\|\max _{\varepsilon_{j}= \pm 1}\left|\sum_{j=1}^{N} \varepsilon_{j} \phi_{j} x_{j}\right|_{X}\right\|_{L^{\infty}(\mathbf{T})} .
$$

Proof. We apply Lemma 2.2 with $f_{j}=\phi_{j} x_{j}$ and $I_{j k}=\{k\}$, and consider the limit where the collection $I_{j k}$ covers all of $\mathbb{Z}$ for each $j$. Then (2.1) reads

$$
\left\|\mathbb{E}\left|\sum_{j, k} \mathbf{r}_{j k} \hat{\phi}_{j}(k) x_{j}\right|_{X}\right\|_{L^{1}(\mathbf{T})} \leq C\left\|\max _{\varepsilon_{j}= \pm 1}\left|\sum_{j} \varepsilon_{j} \phi_{j} x_{j}\right|_{X}\right\|_{L^{\infty}(\mathbf{T})} .
$$

We already know that $X$ is a UMD space, hence has finite cotype. In this situation, the expectations of norms of random sums of vectors of $X$ with Rademacher coefficients $\mathbf{r}_{j}$ are comparable to similar expression with independent standard complex Gaussian random variables $\gamma_{j}$ in place of $\mathbf{r}_{j}$; see e.g. [9], 12.27. Thus

$$
\left\|\mathbb{E}\left|\sum_{j} \sum_{k} \mathbf{r}_{j k} \hat{\phi}_{j}(k) x_{j}\right|_{X}\right\|_{L^{1}(\mathbf{T})} \approx\left\|\mathbb{E}\left|\sum_{j}\left(\sum_{k} \gamma_{j k} \hat{\phi}_{j}(k)\right) x_{j}\right|_{X}\right\|_{L^{1}(\mathbf{T})}
$$

Observe that $\Gamma_{j}:=\sum_{k} \gamma_{j k} \hat{\phi}_{j}(k)$ are centered independent Gaussian random variable with variances $\mathbb{E}\left|\Gamma_{j}\right|^{2}=\sum_{k}\left|\hat{\phi}_{j}(k)\right|^{2}=\left\|\phi_{j}\right\|_{L^{2}(\mathbf{T})}^{2}$. Hence

$$
\left\|\mathbb{E}\left|\sum_{j} \sum_{k} \mathbf{r}_{j k} \hat{\phi}_{j}(k) x_{j}\right|_{X}\right\|_{L^{1}(\mathbf{T})} \approx\left\|\mathbb{E}\left|\sum_{j} \gamma_{j}\left\|\phi_{j}\right\|_{L^{2}(\mathbf{T})} x_{j}\right|_{X}\right\|_{L^{1}(\mathbf{T})} .
$$

After changing the $\gamma_{j}$ back to $\mathbf{r}_{j}$, we have proved the assertion.

We are finally in a position to establish that the property $L P R_{\infty, 1}^{=}(\mathbf{T})$ implies type 2 . We apply the Corollary with $\phi_{j}=1_{[(j-1) / N, j / N)}$ and $x_{j}$ unit vectors in $X$. The result is

$$
\mathbb{E}\left|\sum_{j=1}^{N} \mathbf{r}_{j} x_{j}\right|_{X} \leq C N^{1 / 2}, \quad\left|x_{1}\right|_{X}=\ldots=\left|x_{N}\right|_{X}=1 .
$$

This is the type 2 estimate for vectors of equal length. However, by an observation made by R. C. James [14], this already implies the full type 2 condition, and we are done.

\section{Proof of the Littlewood-Paley estimate for equal intervals. Case of THE Unit CIRCLE}

Our vector-valued proof follows the approach of Rubio de Francia [19] from the scalarvalued case, although he dealt with the analogous estimate on $\mathbb{R}$ instead of $\mathbf{T}$. The first step consists in replacing the spectral projections $S_{I}$ by some nicer approximations. Thus, let $m_{j}$ be functions such that $m_{j} \mid I_{j} \equiv 1$, and let

$$
T_{m_{j}} f:=\sum_{k \in \mathbb{Z}} m_{j}(k) \hat{f}(k) e_{k}
$$


be the corresponding Fourier multiplier operators. Then

$$
\sum_{j} \mathbf{r}_{j} S_{I_{j}} f=\sum_{j} \mathbf{r}_{j} S_{I_{j}} T_{m_{j}} f .
$$

Lemma 3.1. Let $X$ be a UMD space. Let $p \in(1, \infty)$ and $w \in A_{p}$. Then, for any family of non-intersecting subintervals $I_{j}$ of $\mathbb{Z}$ (of arbitrary lengths) and any family $\left\{g_{j}\right\}$ of functions in $L_{X}^{p}(\mathbf{T})$, there holds

$$
\left\|\mathbb{E}\left|\sum_{j} \mathbf{r}_{j} S_{I_{j}} g_{j}\right|_{X}\right\|_{L^{p}(\mathbf{T}, w)} \leq C\left\|\mathbb{E}\left|\sum_{j} \mathbf{r}_{j} g_{j}\right|_{X}\right\|_{L^{p}(\mathbf{T}, w)}
$$

and also

$$
\left\|\mathbb{E}\left|\sum_{j} \mathbf{r}_{j} S_{I_{j}} g_{j}\right|_{X}\right\|\left\|_{\exp L(\mathbf{T})} \leq C\right\| \mathbb{E}\left|\sum_{j} \mathbf{r}_{j} g_{j}\right|_{X} \|_{L^{\infty}(\mathbf{T})} .
$$

Proof. We write out the proof of (3.2) and indicate the changes for (3.1). We first note that each $S_{I_{j}}$ is a linear combination of two translated Hilbert transforms, $S_{I_{j}}=\frac{i}{2}\left[e_{-a_{j}} H e_{a_{j}}-\right.$ $\left.e_{-b_{j}} H e_{b_{j}}\right]$. By the triangle inequality, it suffices to treat the first term. By the contraction principle (applied for $p=1$ ),

$$
\begin{aligned}
\left\|\mathbb{E}\left|\sum_{j} \mathbf{r}_{j} e_{-a_{j}} H\left(e_{a_{j}} g_{j}\right)\right|_{X}\right\|_{\exp L(\mathbf{T})} & \leq 2\left\|\mathbb{E}\left|\sum_{j} \mathbf{r}_{j} H\left(e_{a_{j}} g_{j}\right)\right|_{X}\right\|_{\exp L(\mathbf{T})} \\
& =2\left\|H\left(\sum_{j} \mathbf{r}_{j} e_{a_{j}} g_{j}\right)\right\|_{\exp L_{\operatorname{Rad} X}(\mathbf{T})},
\end{aligned}
$$

where $\operatorname{Rad} X$ is the Banach space formed as the closure of the linear span of the functions $\mathbf{r}_{j} x, x \in X$, in $L_{X}^{1}(\Omega)$. This is again a UMD space (since by Kahane's inequality, the same space with an equivalent norm would be obtained by taking the closure in $L_{X}^{2}(\Omega)$ ). The same lines are also true with the weighted $L^{p}$ space in place of $\exp L$.

We now make use of the John-Nirenberg inequality (valid in arbitrary Banach spaces) and the $L^{\infty}-B M O$-boundedness of the Hilbert transform (in UMD spaces) to continue the estimation

$$
\begin{aligned}
\lesssim\left\|H\left(\sum_{j} \mathbf{r}_{j} e_{a_{j}} g_{j}\right)\right\|_{B M O_{\mathrm{Rad} X}(\mathbf{T})} & \lesssim\left\|\sum_{j} \mathbf{r}_{j} e_{a_{j}} g_{j}\right\|_{L_{\mathrm{Rad} X}^{\infty}(\mathbf{T})} \\
& =\left\|\mathbb{E}\left|\sum_{j} \mathbf{r}_{j} e_{a_{j}} g_{j}\right|_{X}\right\|_{L^{\infty}(\mathbf{T})} \leq 2\left\|\mathbb{E}\left|\sum_{j} \mathbf{r}_{j} g_{j}\right|_{X}\right\|_{L^{\infty}(\mathbf{T})} .
\end{aligned}
$$

In the weighted case, we may directly use the boundedness of $H$ in $L_{X}^{p}(\mathbf{T}, w)$, and the rest is similar with $L^{p}(\mathbf{T}, w)$ in place of $L^{\infty}(\mathbf{T})$.

With Lemma 3.1, our task is reduced to proving that

$$
\left\|\mathbb{E}\left|\sum_{j} \mathbf{r}_{j} T_{m_{j}} f\right|_{X}\right\|_{L^{p}(\mathbf{T}, w)} \leq C\|f\|_{L_{X}^{p}(\mathbf{T}, w)}
$$


for appropriate smooth majorants $m_{j}$ of $1_{I_{j}}$. To describe our choice of the $m_{j}$, we recall the definition of the de la Vallée Poussin kernel (see e.g. [15])

$$
V_{n}=2 K_{2 n+1}-K_{n},
$$

where $K_{n}$ is the Fejér kernel

$$
K_{n}(t)=\sum_{k=-n}^{n}\left(1-\frac{|k|}{n+1}\right) e_{k}(t)=\frac{1}{n+1}\left(\frac{\sin (n+1) \pi t}{\sin \pi t}\right)^{2} .
$$

By applying the estimates $|\sin (n+1) x| \leq(n+1)|\sin x|$ for $x \in \mathbb{R}$ (use the Euler formulas) and $\sin x \geq 2 x / \pi$ for $0 \leq x \leq \pi / 2$, one gets

$$
K_{n}(t) \leq \min \left(n+1, \frac{1}{4(n+1) t^{2}}\right), \quad|t| \leq 1 / 2 .
$$

Thus $\hat{V}_{n-1}(k)=1$ for $|k| \leq n$, and

$$
\left|V_{n-1}(t)\right| \leq C \min \left(n, \frac{1}{n t^{2}}\right), \quad|t| \leq 1 / 2 .
$$

Let $L$ stand for the common length of our intervals $I_{j}$. For a fixed $j$, there are $L+1$ consecutive integers $k$ such that $\hat{V}_{L-1}(\cdot+k)=1$ on $I_{j}$. Let us pick such a $k$ which is also divisible by $L$, and write $k=L k_{j}$. For uniqueness, let us take the smaller $k$ (and then the smaller $k_{j}$ ) if two possibilities exist. Then the mapping $I_{j} \mapsto k_{j}$ is one-to-one, and by reindexing the intervals if necessary, we may assume that $k_{j}=j$, where $j$ ranges over an index set $J \subset \mathbb{Z}$. Thus we take

$$
m_{j}=\hat{V}_{L-1}(\cdot+L j), \quad j \in J,
$$

so that

$$
\sum_{j \in J} \mathbf{r}_{j} T_{m_{j}} f(t)=\sum_{j \in J} \mathbf{r}_{j} \int_{\mathbf{T}} e_{-L j}(y) V_{L-1}(y) f(t-y) \mathrm{d} y .
$$

For a fixed $t \in \mathbf{T}$, we want to estimate the norm of this object in $\operatorname{Rad} X$, which we now equip with the norm of $L_{X}^{2}(\Omega)$ for convenience. Since $X$, as a UMD space, is $B$-convex, we have $(\operatorname{Rad} X)^{*} \approx \operatorname{Rad} X^{*}$, see e.g. [13], Section 3. In particular, one has

$$
\left\|\sum_{j \in J} \mathbf{r}_{j} T_{m_{j}} f(t)\right\|_{\operatorname{Rad} X} \approx \sup \left\{\left|\sum_{j \in J}\left\langle\lambda_{j}, T_{m_{j}} f(t)\right\rangle\right|: \lambda_{j} \in X^{*},\left\|\sum_{j \in J} \mathbf{r}_{j} \lambda_{j}\right\|_{\operatorname{Rad} X^{*}} \leq 1\right\} .
$$

In what follows, we put $\lambda_{j}=0$ for $j \notin J$, which allows us to extend the summation to all indices $j \in \mathbb{Z}$.

We now manipulate the duality pairing appearing above:

$$
\begin{aligned}
G_{\lambda} f(t):=\sum_{j}\left\langle\lambda_{j}, T_{m_{j}} f(t)\right\rangle & =\int_{\mathbf{T}} V_{L-1}(y)\left\langle\sum_{j} e_{-L j}(y) \lambda_{j}, f(t-y)\right\rangle \mathrm{d} y \\
& =: \int_{\mathbf{T}} V_{L-1}(y)\left\langle g_{\lambda}(L y), f(t-y)\right\rangle \mathrm{d} y
\end{aligned}
$$


where $g_{\lambda}$ is an $X^{*}$-valued function on $\mathbb{R}$ of period 1 ,

$$
g_{\lambda}(y)=\sum_{j \in \mathbb{Z}} e_{-j}(y) \lambda_{j}
$$

Lemma 3.2. Let $X$ have type 2. Then

$$
\left\|g_{\lambda}\right\|_{L_{X^{*}}^{2}(\mathbf{T})} \leq C\left(\mathbb{E}\left|\sum_{j \in \mathbb{Z}} \mathbf{r}_{j} \lambda_{j}\right|_{X^{*}}^{2}\right)^{1 / 2} .
$$

Proof. Of course, we make use of duality:

$$
\left\|g_{\lambda}\right\|_{L_{X^{*}}^{2}(\mathbf{T})}=\sup \left\{\left|\int_{\mathbf{T}}\left\langle g_{\lambda}(y), \phi(y)\right\rangle \mathrm{d} y\right|:\|\phi\|_{L_{X}^{2}(\mathbf{T})} \leq 1\right\},
$$

where

$$
\begin{gathered}
\left|\int_{\mathbf{T}}\left\langle g_{\lambda}(y), \phi(y)\right\rangle \mathrm{d} y\right|=\left|\int_{\mathbf{T}}\left\langle\sum_{j} e_{-j}(y) \lambda_{j}, \sum_{k} e_{k}(y) \hat{\phi}(k)\right\rangle \mathrm{d} y\right|=\left|\sum_{j}\left\langle\lambda_{j}, \hat{\phi}(j)\right\rangle\right| \\
=\left|\mathbb{E}\left\langle\sum_{j} \mathbf{r}_{j} \lambda_{j}, \sum_{k} \mathbf{r}_{k} \hat{\phi}(k)\right\rangle\right| \leq\left(\mathbb{E}\left|\sum_{j} \mathbf{r}_{j} \lambda_{j}\right|_{X^{*}}^{2}\right)^{1 / 2}\left(\mathbb{E}\left|\sum_{k} \mathbf{r}_{k} \hat{\phi}(k)\right|_{X}^{2}\right)^{1 / 2} .
\end{gathered}
$$

Now finally we employ the assumed type 2 property of $X$. By a result of P. G. Dodds and F. A. Sukochev ([10], Theorem 1.3), this guarantees that

$$
\left(\mathbb{E}\left|\sum_{k} \mathbf{r}_{k} \hat{\phi}(k)\right|_{X}^{2}\right)^{1 / 2} \leq C\left\|\sum_{k} e_{k} \hat{\phi}(k)\right\|_{L_{X}^{2}(\mathbf{T})}=C\|\phi\|_{L_{X}^{2}(\mathbf{T})},
$$

which completes the proof.

Now we can continue with the expression (3.5). We start by estimating the de la Vallée Poussin kernel according to (3.4):

$$
\begin{aligned}
\left|G_{\lambda} f(t)\right| & \leq \int_{\mathbf{T}}\left|V_{L-1}(y)\right|\left|\left\langle g_{\lambda}(L y), f(t-y)\right\rangle\right| \mathrm{d} y \\
& \leq\left(\int_{|y| \leq 1 / L} C L+\sum_{k=1}^{\infty} \int_{2^{k-1} / L<|y| \leq 2^{k} / L} C L 2^{-2 k}\right)\left|g_{\lambda}(L y)\right|_{X^{*}}|f(t-y)|_{X} \mathrm{~d} y \\
& \leq C \sum_{k=0}^{\infty} 2^{-k}\left(\frac{L}{2^{k}} \int_{|y| \leq 2^{k} / L}\left|g_{\lambda}(L y)\right|_{X^{*}}^{2} \mathrm{~d} y\right)^{1 / 2}\left(\frac{L}{2^{k}} \int_{|y| \leq 2^{k} / L}|f(t-y)|_{X}^{2} \mathrm{~d} y\right)^{1 / 2} \\
& \leq C \sum_{k=0}^{\infty} 2^{-k}\left(\frac{L}{2^{k}} 1_{\left[-2^{k} / L, 2^{k} / L\right]} *|f|_{X}^{2}(t)\right)^{1 / 2} \leq C\left(M|f|_{X}^{2}(t)\right)^{1 / 2}
\end{aligned}
$$

where Lemma 3.2, and the condition that $\left\|\sum \mathbf{r}_{j} \lambda_{j}\right\|_{\operatorname{Rad} X^{*}} \leq 1$, were employed in the secondto-last step, while the last estimate is clear from the definition of the Hardy-Littlewood maximal function $M$.

For $p>2$, the estimate (3.3) now follows from the well-known boundedness of $M$ in $L^{q}(\mathbf{T}, w)$ for $w \in A_{q}$ and $q=p / 2>1$; the case $p=\infty$ is of course trivial. For $p=2$, we 
need to stop the preceding computation before estimating by the maximal function, and then observe that the convolution operators with functions $|J|^{-1} 1_{J}$ are uniformly bounded on $L^{1}(\mathbf{T}, w)$ for $w \in A_{2 / 2}=A_{1}$.

It might be interesting to note that out of the two key steps of the proof, Lemma 3.1 only employed the UMD assumption, whereas Lemma 3.2 only used type 2 . Indeed the proof shows that a variant of the $L P R_{p}^{=}(\mathbf{T})$ estimate, with the sharp spectral projections replaced by those of de la Vallée Poussin type, is true in all Banach spaces with type 2.

\section{Proof of Theorem 1.2.}

Lemma 4.1. For any Banach space $X$, the following statements are equivalent

(i) Inequality (1.3) holds for families of disjoint intervals of the same length in $\mathbb{Z}$.

(ii) Inequality (1.4) holds for families of disjoint intervals of the same length in $\mathbb{R}$.

Proof. The proof of $(i i) \Longrightarrow(i)$ can be got by following the proof of Theorem 2.5 in [11].

Now let us assume $(i)$, and let us prove $(i i)$. We will use the fact that the discrete partial Fourier sums (1.1) are Riemann sums of their continuous analogues (1.5). Take a finite number of non-intersecting intervals $\left\{J_{j}\right\}_{j=1}^{m}$ in $\mathbb{R}$ of equal lengths. Let us show first that for any $A>0$ and any $f \in L_{X}^{p}([-A, A])$,

$$
\left\|\mathbb{E} \sum_{j=1}^{m} \mathbf{r}_{j} S_{J_{j}}^{\mathbb{R}} f\right\|_{L_{X}^{p}([-A, A])} \leq C\|f\|_{L_{X}^{p}([-A, A])} .
$$

We shall give an approximation argument. By the assumption $(i)$,

$$
\left\|\mathbb{E}\left|\sum_{j} \mathbf{r}_{j} S_{I_{j}} f\right|_{X}\right\|_{L^{p}([-1 / 2,1 / 2])} \leq C\|f\|_{L_{X}^{p}([-1 / 2,1 / 2])}
$$

for any $f \in L_{X}^{p}([-1 / 2,1 / 2])$ and for any finite sequence $\left\{I_{j}\right\}$ of subintervals of $\mathbb{Z}$ of the same length. First we apply rescaling to (4.2). Given a function $f$ and real $\ell>0$, we define a rescaled function $f_{\ell}(x)=\frac{1}{\ell} f\left(\frac{x}{\ell}\right)$. One has

$$
\widehat{\left(f_{1 / \ell}\right)}(\lambda)=\hat{f}\left(\frac{\lambda}{\ell}\right) \text {. }
$$

Given a finite subinterval $I$ of the discrete set $\ell^{-1} \mathbb{Z}$, define

$$
S_{I}^{(\ell)} f(x)=\frac{1}{\ell} \sum_{\lambda \in I} \hat{f}(\lambda) e^{i 2 \pi \lambda x} .
$$

Then $\ell I$ is a subinterval in $\mathbb{Z}$, and it is easy to see that

$$
S_{I}^{(\ell)} f(x)=\left(S_{\ell I}\left(f_{1 / \ell}\right)\right)_{\ell}(x) .
$$

We get from (4.2) the inequality

$$
\left\|\mathbb{E}\left|\sum_{j=1}^{m} \mathbf{r}_{j} S_{I_{j}}^{(\ell)} f\right|_{X}\right\|_{L_{X}^{p}([-\ell / 2, \ell / 2])} \leq C\|f\|_{L_{X}^{p}([-\ell / 2, \ell / 2])}
$$


for functions $f \in L_{X}^{p}([-\ell / 2, \ell / 2])$ and subintervals $I_{j}$ of $\ell^{-1} \mathbb{Z}$, with the same constant $C$ as in (4.2).

To prove (4.1), take any $\ell>\max \left(2 A, 1 / 2\left|J_{j}\right|\right)$, so that $[-\ell / 2, \ell / 2] \supset[-A, A]$. We extend our function $f \in L_{X}^{p}([-A, A])$ to a function in $L_{X}^{p}(\mathbb{R})$ by putting $f \equiv 0$ on $\mathbb{R} \backslash[-A, A]$. It is easy to find subintervals $I_{j, \ell} \subset J_{j} \cap \ell^{-1} \mathbb{Z}$ with the following two properties:

(1) For a fixed $\ell$, the intervals $I_{j, \ell}$ are disjoint and have the same length;

(2) $\left|J_{j}\right|-\frac{2}{\ell} \leq\left|I_{j, \ell}\right|<\left|J_{j}\right|-\frac{1}{\ell}$.

One easily gets from (2) an estimate

$$
\left|\int_{J_{j}} g(\lambda) d \lambda-\frac{1}{\ell} \sum_{\lambda \in I_{j, \ell}} g(\lambda)\right|_{X} \leq \frac{\left|J_{j}\right|}{2 \ell} \max _{\lambda \in J_{j}}\left|\frac{d g(\lambda)}{d \lambda}\right|_{X}+\frac{2}{\ell} \max _{\lambda \in J_{j}}|g(\lambda)|_{X}
$$

which holds for any $g \in C_{X}^{1}\left(J_{j}\right)$. By putting here $g(\lambda)=\hat{f}(\lambda) e^{i 2 \pi \lambda x}$, one gets

$$
\max _{x \in[-A, A]}\left|\mathbb{E} \sum_{j=1}^{m} \mathbf{r}_{j} S_{J_{j}}^{\mathbb{R}} f(x)-\mathbb{E} \sum_{j=1}^{m} \mathbf{r}_{j} S_{I_{j, \ell}}^{(\ell)} f(x)\right|_{X} \leq \frac{K}{\ell}\|f\|_{L_{X}^{1}([-A, A])} \rightarrow 0 \text { as } \ell \rightarrow \infty ;
$$

the constant $K$ here depends on the intervals $J_{j}$ and on $A$, but not on $\ell$. By (4.3),

$$
\left\|\mathbb{E}\left|\sum_{j=1}^{m} \mathbf{r}_{j} S_{I_{j, \ell}^{(\ell)}}^{(\ell)}\right|_{X}\right\|_{L_{X}^{p}([-A, A])} \leq C\|f\|_{L_{X}^{p}([-A, A])} .
$$

Now (4.1) is obtained by passing to the limit as $\ell$ goes to $\infty$ and taking into account (4.4).

Next, one can pass to the limit in (4.1) as $A \rightarrow \infty$ to get (ii).

Now we can give the proof of Theorem [1.2, By using Theorem 1.1 and Lemma 4.1 we have that if a Banach space $X$ is a UMD space with type 2 , then $X$ has $L P R_{p}^{=}(\mathbb{R})$ for all $p \in[2, \infty)$. In this case by using Lemma 3.1 and the parallel estimate to (3.3) developed in the original paper [19] we get that $X$ has $\operatorname{LPR}_{p}^{=}(\mathbb{R}, w)$ for all $p \in[2, \infty)$ and all $w \in A_{p / 2}$. For the converse implications we just use Lemma 4.1 and Theorem 1.1 .

\section{5. $L^{\infty}-$ BMO UNBOUNDEDNESS}

One could ask whether in the $L P R_{\infty}^{=}(\mathbf{T})$ property, the $\exp L$ norm can be replaced by the BMO norm. Here we show that it is not the case; moreover, even in the one-interval inequality

$$
\left\|S_{I} f\right\|_{\mathrm{BMO}(\mathbf{T})} \leq C\|f\|_{L^{\infty}(\mathbf{T})}
$$

$C$ cannot be chosen independently on the subinterval $I \subset \mathbb{Z}$.

Indeed, take the interval $I=[0, n) \subset \mathbb{Z}$, it is well known that

$$
S_{[0, n)} f=\frac{i}{2}\left(H f-e_{n} H\left(e_{-n} f\right)\right),
$$

where $H=-i\left[S_{[0, \infty)}-S_{(-\infty, 0)}\right]$ is the Hilbert transform on $\mathbf{T}$, and we recall our notation $e_{n}(x)=e^{i 2 \pi n x}$. As $H$ is bounded from $L^{\infty}(\mathbf{T})$ into $\operatorname{BMO}(\mathbf{T})$, inequality (5.1) is equivalent 
to the existence of a constant $C$ such that for any $n \in \mathbb{Z}$, we have

$$
\left\|e_{n} H\left(e_{-n} f\right)\right\|_{\operatorname{BMO}(\mathbf{T})} \leq C\|f\|_{L^{\infty}(\mathbf{T})} .
$$

By choosing $f=e_{n} g$ with $g \in L^{\infty}(\mathbf{T})$ we infer that

$$
\left\|e_{n} H(g)\right\|_{\mathrm{BMO}(\mathbf{T})} \leq C\|g\|_{L^{\infty}(\mathbf{T})},
$$

where $C$ should not depend on $n$. Choose a function $g \in L^{\infty}(\mathbf{T})$ such that $H g \in \operatorname{BMO}(\mathbf{T}) \backslash$ $L^{\infty}(\mathbf{T})$, then next Lemma shows that a uniform estimate as in (5.3) cannot hold.

Lemma 5.1. Suppose $f \in \operatorname{BMO}(\mathbf{T})$ satisfies $\sup _{n \in \mathbb{Z}}\left\|e_{n} f\right\|_{\mathrm{BMO}(\mathbf{T})} \leq C<\infty$. Then $f \in$ $L^{\infty}(\mathbf{T})$.

Proof. We interpret $f$ as a 1-periodic function on $\mathbb{R}$. For an interval $K$ of the real line of length less than 1 and a 1 -periodic function $g$ on $\mathbb{R}$, put $g_{K}=\frac{1}{|K|} \int_{K} g$. One has the estimates

$$
\frac{1}{|K|} \int_{K}|f|=\frac{1}{|K|} \int_{K}\left|f e_{n}\right| \leq \frac{1}{|K|} \int_{K}\left|f e_{n}-\left(f e_{n}\right)_{K}\right|+\left|\left(f e_{n}\right)_{K}\right| \leq C+C=2 C
$$

for a sufficiently large $|n|$, due to the Riemann-Lebesgue lemma. It follows that $M|f|(x) \leq$ $2 C$ for any $x \in \mathbf{T}$, and therefore $\|f\|_{L^{\infty}(\mathbb{R})} \leq 2 C$.

The conclusion of the above is that an $L^{\infty}-\mathrm{BMO}$ estimate in Theorem 1.1 has no sense.

\section{REFERENCES}

[1] Berkson, E., Gillespie, T. A., Torrea, J. L., Vector Valued Transference. Functional Space Theory and its applications (ed. Peide Liu). Proceedings of International Conference \& 13th Academic Symposium in China. 1-27. Wuhan 2003.

[2] Blasco, O., Hardy spaces of vector-valued functions: duality. Trans. Amer. Math. Soc. 308 (1988), no. 2, 495-507.

[3] Bourgain, J., Some remarks on Banach spaces in which martingale difference sequences are unconditional. Ark. Mat. 21 (1983), no. 2, 163-168.

[4] Bourgain, J., Vector-valued singular integrals and the $H^{1}$-BMO duality. Probability theory and harmonic analysis (Cleveland, Ohio, 1983), 1-19, Monogr. Textbooks Pure Appl. Math., 98, Dekker, New York, 1986.

[5] Burkholder, D. L., A geometric condition that implies the existence of certain singular integrals of Banach-space-valued functions. Conference on harmonic analysis in honor of Antoni Zygmund, Vol. I, II (Chicago, Ill., 1981), 270-286, Wadsworth Math. Ser., Wadsworth, Belmont, CA, 1983.

[6] Carleson, L., On the Littlewood-Paley theorem, Mittag-Leffler Inst. Report, Djursholm, 1967.

[7] Córdoba, A., Some remarks on the Littlewood-Paley theory. Proceedings of the Seminar on Harmonic Analysis (Pisa, 1980). Rend. Circ. Mat. Palermo (2) 1981, suppl. 1, 75-80.

[8] Cowling, M.; Tao, T. Some light on Littlewood-Paley theory. Math. Ann. 321 (2001), no. 4, 885-888.

[9] Diestel, J., Jarchow, H., Tonge, A., Absolutely summing operators. Cambridge Studies in Advanced Mathematics, 43. Cambridge University Press, Cambridge, 1995. xvi+474 pp.

[10] Dodds, P. G., Sukochev, F. A., RUC-decompositions in symmetric operator spaces. Integral Equations Operator Theory 29 (1997), no. 3, 269-287.

[11] Gillespie, T. A., Torrea, J. L., Transference of a Littlewood-Paley-Rubio inequality and dimension free estimates. Rev. Un. Mat. Argentina 45 (2004), no. 1, 1-6 (2005).

[12] Hytönen, T. P., Potapov, D. Vector-valued multiplier theorems of Coifman-Rubio de Francia-Semmes type. Arch. Math. (Basel) 87 (2006), no. 3, 245-254. 
[13] Hytönen, T., Weis, L., Singular convolution integrals with operator-valued kernel. Math. Z. 255 (2007), no. $2,393-425$.

[14] James, R. C., Nonreflexive spaces of type 2. Israel J. Math. 30 (1978), no. 1-2, 1-13.

[15] Katznelson, Y., An introduction to harmonic analysis. Second corrected edition. Dover Publications, Inc., New York, 1976. xiv+264 pp.

[16] Kislyakov, S. V., Parilov, D. V., On the Littlewood-Paley thoerem for arbitrary intervals, Zapiski Nauchn. Sem. POMI 327 (2005), 98-114; Engl. transl. in J. Math. Sci. 139, No. 2 (2006), 6417-6424.

[17] Kunstmann, P. C., Weis, L., Maximal $L_{p}$-regularity for parabolic equations, Fourier multiplier theorems and $H^{\infty}$-functional calculus. Functional analytic methods for evolution equations, 65-311, Lecture Notes in Math., 1855, Springer, Berlin, 2004.

[18] Lacey, M. T. Issues related to Rubio de Francia's Littlewood-Paley inequality. NYJM Monographs, 2. Albany, NY, 2007. 36 pp.

[19] Rubio de Francia, J. L., Estimates for some square functions of Littlewood-Paley type. Publ. Sec. Mat. Univ. Autònoma Barcelona 27 (1983), no. 2, 81-108.

[20] Rubio de Francia, J. L., A Littlewood-Paley inequality for arbitrary intervals. Rev. Mat. Iberoamericana 1 (1985), no. 2, 1-14.

Department of Mathematics and Statistics, University of Helsinki, Gustaf Hällströmin KATU 2B, 00014 Helsinki, Finland

E-mail address: tuomas.hytonen@helsinki.fi

Departamento de Matemáticas, Universidad Autónoma de Madrid, Cantoblanco, 28049 MADRID, SPAIN

E-mail address: joseluis.torrea@uam.es

E-mail address: dmitry.yakubovich@uam.es 